\title{
Possible healthcare-associated transmission as a cause of secondary infection and population structure of Staphylococcus aureus isolates from two wound treatment centres in Ghana
}

\author{
G. Kpeli ${ }^{1,2,3}$, I. Darko Otchere', A. Lamelas ${ }^{3,6}$, A. L. Buultjens ${ }^{4}$, D. Bulach ${ }^{4,5}$, S. L. Baines ${ }^{4}$, T. Seemann ${ }^{4,5}$, S. Giulieri ${ }^{4}$, Z. Nakobu', \\ S. Y. Aboagye', E. Owusu-Mireku', G. Pluschke ${ }^{2,3}$, T. P. Stinear ${ }^{4}$ and D. Yeboah-Manu' \\ I) Noguchi Memorial Institute for Medical Research, Accra, Ghana, 2) Swiss Tropical and Public Health Institute, 3) University of Basel, Basel, \\ Switzerland, 4) Doherty Applied Microbial Genomics, Department of Microbiology and Immunology, Doherty Institute for Infection and Immunity, University of \\ Melbourne, Melbourne, 5) Victorian Life Sciences Computation Initiative, University of Melbourne, Parkville, VIC, Australia and 6) Red de Estudios Moleculares \\ Avanzados, Instituto de Ecología, A.C, Carretera antigua a Coatepec 35I, El Haya Xalapa, Veracruz, Mexico
}

\section{Abstract}

We have previously shown that secondary infections of Buruli ulcer wounds were frequently caused by Staphylococcus aureus. To gain understanding into possible routes of secondary infection, we characterized S. aureus isolates from patient lesions and surrounding environments across two Ghanaian health centres. One hundred and one S. aureus isolates were isolated from wounds $(n=93,92.1 \%)$ and the hospital environment $(n=8,7.9 \%)$ and characterized by the spa gene, mecA and the Panton-Valentine leucocidin toxin followed by spa sequencing and whole genome sequencing of a subset of 49 isolates. Spa typing and sequencing of the spa gene from 91 isolates identified 29 different spa types with t355 (STI52), tl 86 (ST88), and t346 dominating. Although many distinct strains were isolated from both health centres, genotype clustering was identified within centres. In addition, we identified a cluster consisting of isolates from a healthcare worker, patients dressed that same day and forceps used for dressing, pointing to possible healthcare-associated transmission. These clusters were confirmed by phylogenomic analysis. Twenty-four $(22.8 \%)$ isolates were identified as methicillin-resistant $S$. aureus and lukFS genes encoding Panton-Valentine leucocidin were identified in 67 (63.8\%) of the isolates. Phenotype screening showed widespread resistance to tetracycline, erythromycin, rifampicin, amikacin and streptomycin. Genomics confirmed the widespread presence of antibiotic resistance genes to $\beta$-lactams, chloramphenicol, trimethoprim, quinolone, streptomycin and tetracycline. Our findings indicate that the healthcare environment probably contributes to the superinfection of Buruli ulcer wounds and calls for improved training in wound management and infection control techniques.

(C) 2016 The Author(s). Published by Elsevier Ltd on behalf of European Society of Clinical Microbiology and Infectious Diseases.

Keywords: Buruli ulcer, health care-associated, infection, spa typing, Staphylococcus aureus, whole genome sequencing Original Submission: 30 April 2016; Revised Submission: 24 June 2016; Accepted: I July 2016

Article published online: 12 July 2016

Corresponding author: D. Yeboah-Manu, Noguchi Memorial Institute for Medical Research, P.O. Box 58I, Legon, Accra, Ghana

E-mail: dyeboah-manu@noguchi.ug.edu.gh

\section{Introduction}

Microbial contamination and colonization of wounds is common to all wounds healing by secondary intention and has been proposed as a precondition to the formation of granulation tissue and stimulation of wound healing [I]. Wounds can be infected through three main sources; the surrounding skin, endogenous sources such as the nasal mucosa, gastrointestinal tract and genitourinary tract and the wider environment. Within a healthcare facility, the sources of contamination and subsequent infection of a wound may include healthcare workers $(\mathrm{HCW})$, patients and the inanimate environment. Direct contact of a patient with an infected HCW during general care or medical treatment can result in the transmission 
of microorganisms. Indirectly, an infected patient or HCW could touch and contaminate an object, instrument or surface and subsequent contact between the item and a second patient is likely to contaminate that patient leading to infection. Wound microflora is usually polymicrobial [2] comprising organisms such as staphylococci, enterococci, streptococci, facultative Gram-negative bacteria and anaerobic bacteria [3].

Staphylococcus aureus is a notorious opportunistic nosocomial pathogen, and one of the main organisms involved in the infection of chronic wounds [4]. It may be carried asymptomatically by a carrier and transferred from patient to patient. It is estimated that about $25 \%$ of the normal population may be carriers, with higher carriage rates around $50 \%$ in patients with insulin-dependent diabetes, intravenous drug users and patients on dialysis [5].

In a previous study, we identified infection of Buruli ulcer (BU) wounds by bacterial pathogens as a possible cause of wound healing delay among study participants [6]. Several bacterial species were isolated with $S$. aureus and Pseudomonas aeruginosa dominating. Despite the finding that most of the organisms identified were nosocomial pathogens, the possible routes of infection of the lesions were not examined in this study. In seeking to have a better understanding of the possible routes of infection of the lesions, we initiated the current study to characterize $S$. aureus isolated from different sources in two health facilities in Ghana treating BU.

\section{Materials and methods}

\section{Study sites, participants and sample collection}

The study was carried out at the two main BU treatment centres in the $\mathrm{Ga}$ West and $\mathrm{Ga}$ South districts of the Greater Accra Region of Ghana, designated facility A and facility B, respectively. The study involved the analysis of $S$. aureus isolates from wounds of microbiologically confirmed $B U(B U)$ and non$\mathrm{BU}(\mathrm{NBU})$ patients (initially suspected BU cases that were not confirmed by any of the three confirmation methodsZiehl-Neelson microscopy, IS2404 PCR and culture) who received treatment either as inpatients on admission or outpatients at one of the two health centres from October 2010 to February 2014. Lesions of patients were swabbed and the samples were analysed at the Bacteriology Department of the Noguchi Memorial Institute for Medical Research. A total of 173 samples were collected from 162 patients and S. aureus was isolated from 88 samples originating from 76 patients, $6 \mathrm{I}$ of whom were BU patients and I 5 were NBU patients. Of the 61 $\mathrm{BU}$ patients, 56 were outpatients and five were inpatients at the time of sampling, whereas all the NBU patients were outpatients. The five inpatients had been on admission for 4 weeks or more and therefore isolates from these patients could be classified as potentially hospital acquired. To elucidate the sources of infection, between August 2013 and February 2014 the hands of HCW, dressing rooms and wards of the health centres, surfaces of dressing tables, door handles, instruments and equipment as well as dressing solutions and materials were sampled for microbiological analysis. Swab samples were also taken from the palms of nurses and their gloved hands in between dressing of patients and the lesions of the patients they dressed before and after dressing. Swab samples were transported in phosphate buffered saline at $4^{\circ} \mathrm{C}$ to the Noguchi Memorial Institute for Medical Research. Eighty-six samples were collected and analysed and S. aureus was isolated from 13 of them (see Supplementary material, Fig. SI).

\section{Microbiological methods}

The samples were processed and inoculated on blood and mannitol salt agars and incubated for $18-24 \mathrm{~h}$ at $37^{\circ} \mathrm{C}$, after which they were examined. Identification of Staphylococcus species was by colony and microscopic morphology, catalase reaction and the coagulase biochemical test (BD, Franklin Lakes, NJ, USA). The Staphylase Kit, BD BBL ${ }^{\text {TM }}$ Staphyloslide Latex Test (BD, Franklin Lakes, NJ, USA) was used to differentiate the catalase-positive Gram-positive bacteria $S$. aureus from other Staphylococcus species.

\section{Antibiogram of isolated bacteria}

Antibiotic susceptibility testing was determined by the KirbyBauer disc diffusion method according to CLSI guidelines [7]. Susceptibility was determined for the antibiotics; amikacin $(30 \mu \mathrm{g})$, sulphamethoxazole-trimethoprim (23.75 $\mu \mathrm{g} / \mathrm{l} .25 \mu \mathrm{g})$, ampicillin $(10 \mu \mathrm{g})$, tetracycline $(30 \mu \mathrm{g})$, gentamicin $(10 \mu \mathrm{g})$, erythromycin $(15 \mu \mathrm{g})$, cefuroxime $(30 \mu \mathrm{g})$, ceftriaxone $(30 \mu \mathrm{g})$, chloramphenicol $(30 \mu \mathrm{g})$, cefoxitin $(30 \mu \mathrm{g})$, rifampicin $(5 \mu \mathrm{g})$, streptomycin $(10 \mu \mathrm{g})$, vancomycin $(30 \mu \mathrm{g})$, clindamycin $(2 \mu \mathrm{g})$ and cefotaxime $(30 \mu \mathrm{g})$. Cartridges of antibiotics were obtained from Oxoid (Wade Road, Basingstoke, Hampshire, United Kingdom) and BD. Staphylococcus aureus ATCC 25923 was used as reference strain.

\section{Genotyping - traditional molecular typing}

Crude DNA extracts were prepared by boiling and used as template in the PCR. Genetic relatedness of the isolates was determined through amplification of the polymorphic $X$ region of the protein A (spa) gene using the primer pairs spa- I I I3F and spaI5I4R (see Supplementary material, Table SI) [8] and DNA from S. aureus ATCC ${ }^{\circledR} 25923$ as positive control. A total of 95 isolates out of the $10 \mathrm{I}$ were typed using these primers. A number of suspected $S$. aureus strains could not be typed by these primers and were further typed using another set of primers, spaT3-F and spa-I5I7R (see Supplementary material, Table SI). These primers 
were previously described to detect $S$. aureus strains that may have rearrangements in the lgG-binding region of the gene where the forward primer is located, making them untypeable by the original spa primers [9]. Sequencing of the spa gene from positive isolates was outsourced to Macrogen Europe, (Amsterdam, the Netherlands) and sequences were analysed and assigned to spa types using DNAGear [10]. The relationship between strains was investigated using the STADEN package [I I] and a maximum likelihood phylogenetic tree produced in MEGA 5.05 (www.megasoft. net) and visualized in FIGTREE vl.4.2. (http://tree.bio.ed.ac.uk/ software/figtree/).

Genes for mecA and Panton-Valentine leucocidin (PVL) were sought using the primers mecA P4, mecA P7 and pvl-F, pvl-R (see Supplementary material, Table SI) [I2,I3]. A mecA and PVL-positive isolate S. aureus 282-10I from the Statens Serum Institute (Copenhagen, Denmark) kindly provided by Dr Beverly Egyir, was used as positive control. Agr types were determined using the primers pan agr $F$, agr I-R, agr 2-R, agr 3$\mathrm{R}$ and agr 4-R (see Supplementary material, Table SI) [I4]. The PCR contained I $2.5 \mu \mathrm{L}$ HotStarTaq Mastermix (Qiagen, Hilden, Germany), $5 \mu \mathrm{L}$ nuclease-free water, $2 \mu \mathrm{L}$ of each primer and $3.5 \mu \mathrm{L}$ of DNA. Results of mecA, agr and PVL typing were analysed using Microsoft EXCEL.

\section{Whole genome sequencing and analysis}

From the $10 \mathrm{I}$ isolates, a subset of 70 isolates were selected for whole genome sequencing (WGS) made up of 31 methicillinresistant S. aureus (MRSA) isolates and 39 other methicillinsusceptible $S$. aureus (MSSA) isolates spanning the clusters observed from the phylogenetic tree obtained from sequencing of the spa gene. Genomic DNA was extracted with the Qiagen DNeasy Blood and tissue kit (Qiagen) according to the manufacturer's instructions and sequenced using the Illumina MiSeq platform (2× 250 bp sequencing by synthesis chemistry using DNA libraries prepared using Nextera XT (Illumina, San Diego, CA, USA). Resulting sequence reads were processed using a custom bioinformatics analysis pipeline NULLARBOR (nullarbor.pl 0.6; https://github.com/tseemann/nullabor) to de novo assemble and also align read data against the Sa_aus0325 reference genome (S. Giulieri et al., unpublished). NuLLARBOR uses a BLAST-based method to screen contigs from de novo assemblies for the presence of antibiotic resistance genes. Pairwise alignments of core genome single nucleotide polymorphisms (SNPs) were used with FASTTREe [15] to infer maximum likelihood phylogenetic trees using the general time reversible model of nucleotide substitution. Resulting trees were visualized in FIGTREE vI.4.2. The topologies of rooted phylogenetic trees obtained from both spa typing and WGS using Staphylococcus simiae CCM7213 as an outgroup were compared by tanglegram in DENDROSCOPE (v3.4.4) [ 16$]$.
Results

\section{Bacterial isolates}

In all, I0I S. aureus isolates were identified from the sources sampled. Eighty-eight isolates were recovered from 76 patients during routine sampling; 72 (8I.6\%) were from $6 \mathrm{I}$ BU patients and 15 were from NBU wounds. One isolate each was identified from 66 patients and multiple isolates from the other ten patients. Five patients had two isolates from a single lesion collected at one time-point, one patient had two isolates from two lesions, three isolates were identified from a patient with three lesions (patient $A$, one from each), two patients had two isolates sampled at two different time-points during the course of treatment, and one patient with three isolates sampled thrice at different time-points during the course of treatment.

To understand possible route of infection, additional sampling of HCW, working environment and patients being attended to led to isolation of I 3 (I5.1\%) S. aureus. The isolates comprised five from patient lesions, six from equipment (forceps) and one each from the hand of an HCW and a table used for dressing wounds. All of these isolates were from Facility $B$. In total, 53 isolates were from Facility A and 48 isolates were from Facility B.

\section{Population structure of S. aureus and epidemiological association}

Spa typing identified 29 different spa types including 15 (14.9\%) singles. The three dominant spa types ( $\mathrm{t} 355, \mathrm{t} 186$ and $\mathrm{t} 346)$ were found in seven (6.93\%) isolates each (see Supplementary material, Table S2). Thirty-five isolates could not be assigned a spa type. Phylogenetic analysis identified ten different clusters, where a cluster was defined as a group of two or more isolates found on the same branch of the tree (Clusters A-J; Fig. I), which consisted of health-centre-specific clusters and common clusters. Clusters $\mathrm{A}, \mathrm{C}, \mathrm{D}, \mathrm{H}$ and $\mathrm{J}$ were made up of strains from both health centres (Fig. I) with rates of 2:I, I:I, I:I, I:I and I:2 for Facilities $A$ and $B$, respectively. Clusters $B$ and I were made up of strains from Facility $A$ only, whereas seven out of the ten isolates in cluster $G$ were also from Facility $A$. Clusters $E$ and $F$ were made up mainly of strains from Facility $B$ with rates of $I: 3$ and $2: 12$ for Facility A and B, respectively (Fig. I). Within cluster $F$ were isolates cultured from samples taken on the same day from the hand of an HCW and patients dressed consecutively by this worker, isolates from samples taken on a different day from one equipment (forceps) after it had been used on four patients consecutively and two isolates recovered from a patient at two different time-points (weeks 2 and 8) during treatment (Fig. I). This finding led us to conclude that transmission events were ongoing within this health centre. Cluster $G$ was made up mainly of MRSA 
FIG. I. Spa phylogeny showing clusters and relationships between isolates. Maximum likelihood phylogeny of spa gene. The tree was rooted in the midpoint. Numbers in nodes indicate support values in the form of proportions of bootstrap pseudoreplicates. Branches with support values $>55 \%$ are collapsed. A-J = the clusters identified. The greencoloured strains are from Health centre $A$, and the blue ones are from Health centre B. The yellowcoloured circles represent methicillin-resistant Staphylococcus aureus and the violet circles show isolates from a healthcare worker, patients and equipment in Health centre $B$.

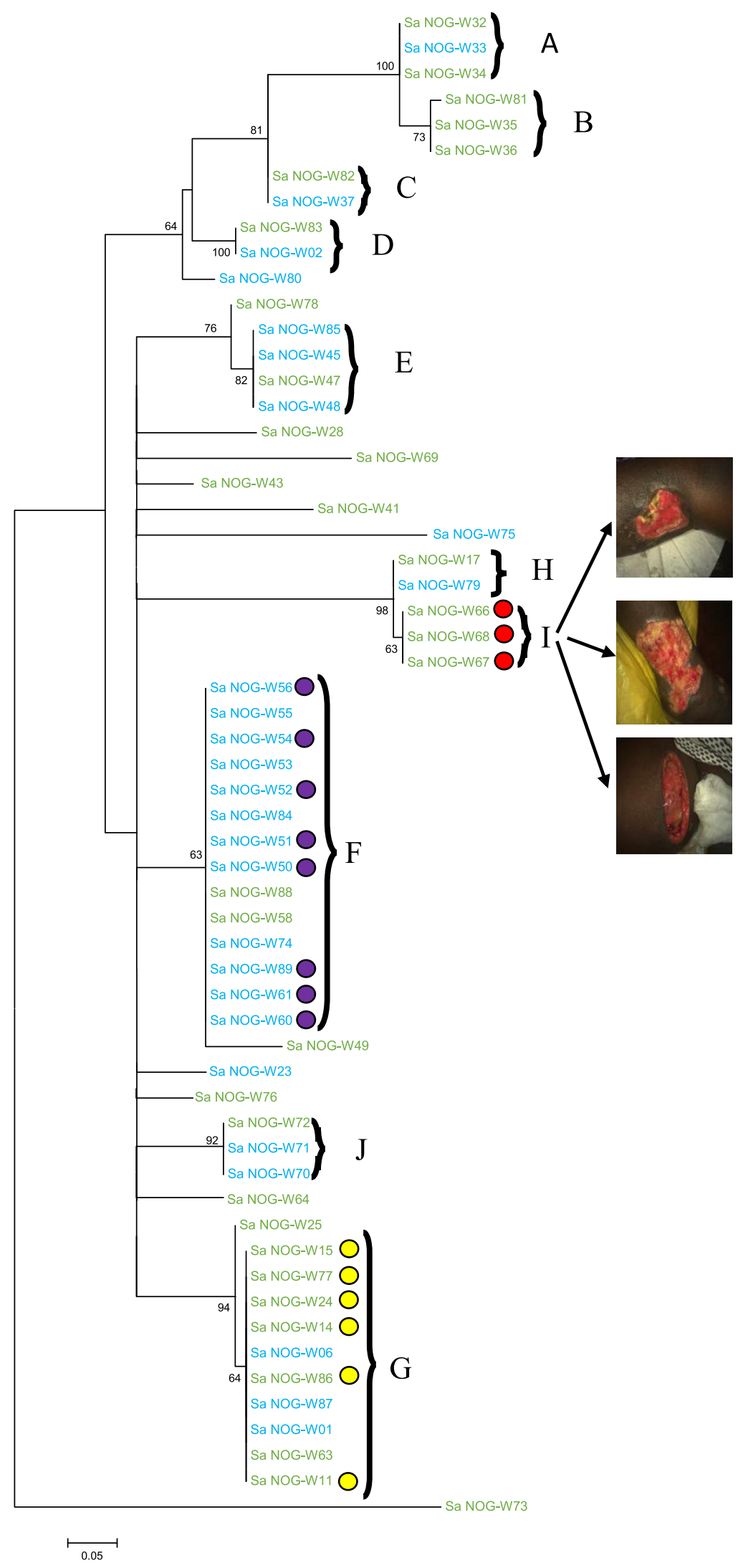


isolates (Fig. I) whereas cluster I was made up of isolates from three different lesions of one patient that had the same spa type (t2500) (Fig. I),, suggesting that the three lesions were infected from a common source, possibly from the patients' microbiota.

\section{Detection of mecA and PVL genes and Agr type}

The PCR screening for the mecA gene identified 31 isolates as MRSA. The genotypes of 24 of these isolates correlated with their antibiogram results; however, mecA was also detected in seven isolates showing susceptible phenotypes. Twenty-one of these were from BU patients, eight were from NBU patients and two were from the environment. Four of the MRSA were isolated from inpatients. The PVL gene was detected in 66 isolates; 5 I from BU patients, 12 from NBU patients and three from the environment. Both mecA and the PVL genes were detected in 20 isolates made up of 14 from BU patients, five from NBU patients and one from the environment. Nineteen (I8.8\%) isolates belonged to agr type I, $23(22.8 \%)$ isolates to agr type 2, $30(29.7 \%)$ isolates to agr type 3, three $(2.9 \%)$ isolates to agr type 4 , and four $(3.9 \%)$ isolates showed bands for both agr types 2 and 3 . Twenty-two (2I.8\%) isolates were nontypeable and possibly agr-defective mutants.

\section{Antibiogram of isolates}

Over $70 \%$ of the isolates were susceptible to amikacin (89; $88 \%$ ) and gentamicin (89; 88\%). Resistance rates $>50 \%$ were recorded against the antibiotics ampicillin (91; 90\%), tetracycline $(57 ; 56.4 \%)$ and chloramphenicol $(67 ; 66 \%)$. Lower resistance rates of $37.6 \%, 39.6 \%$ and $48.5 \%$ were also recorded against the cephalosporins; ceftriaxone, cefotaxime and cefuroxime, respectively, whereas $26 \%$ of the isolates showed reduced susceptibility to vancomycin (Table I). Comparing the antibiograms of MRSA and MSSA isolates, a significant proportion of MRSA isolates were resistant to the tetracycline ( $P$ $<0.05$ ) (Table 2). Resistance to chloramphenicol was equally high among MRSA and MSSA.

\section{TABLE I. Antibiogram of isolates}

\begin{tabular}{|c|c|c|c|}
\hline \multirow[b]{2}{*}{ Antibiotics } & \multicolumn{3}{|c|}{ Antibiogram } \\
\hline & $\begin{array}{l}\text { Sensitive, } \\
n(\%)\end{array}$ & $\begin{array}{l}\text { Intermediate, } \\
\text { n (\%) }\end{array}$ & $\begin{array}{l}\text { Resistant, } \\
\text { n (\%) }\end{array}$ \\
\hline $\begin{array}{l}\text { Amikacin } \\
\text { Cefotaxime } \\
\text { Gentamicin } \\
\text { Tetracycline } \\
\text { Chloramphenicol } \\
\text { Ceftriaxone } \\
\text { Cotrimoxazole } \\
\text { Cefuroxime } \\
\text { Ampicillin } \\
\text { Clindamycin } \\
\text { Cefoxitin } \\
\text { Erythromycin } \\
\text { Rifampicin } \\
\text { Streptomycin } \\
\text { Vancomycin }\end{array}$ & $\begin{array}{l}89(88) \\
50(49.5) \\
89(88) \\
39(38.6) \\
26(26) \\
53(52.5) \\
65(64.3) \\
52(51.5) \\
6(6) \\
52(51.4) \\
66(65) \\
41(41) \\
46(45.5) \\
66(65.3) \\
75(74)\end{array}$ & $\begin{array}{l}7(7) \\
11(10.8) \\
4(4) \\
5(5) \\
8(8) \\
10(10) \\
4(4) \\
0 \\
4(4) \\
18(18) \\
5(5) \\
37(36) \\
9(9) \\
9(9)\end{array}$ & $\begin{array}{l}5(5) \\
40(39.6) \\
8(8) \\
57(56.4) \\
67(66) \\
38(37.6) \\
32(31.6) \\
49(48.5) \\
91(90) \\
31(30.6) \\
30(30) \\
23(23) \\
46(45.5) \\
26(25.7) \\
26(26)\end{array}$ \\
\hline
\end{tabular}

\section{WGS analysis}

During the course of this project we had the opportunity to investigate some of the isolates further by WGS. From an initial 70 isolates subjected to WGS, 21 were of low read-coverage and excluded from analysis. We first inferred multilocus sequence typing data from the WGS data. The 49 isolates belonged to 12 different sequence types (ST) with STI5 (I3 isolates) and ST88 (II isolates) dominating. The remainder belonged to STI (one isolate), ST5 (six isolates), ST6 (one isolate), ST72 (two isolates), STI2I (two isolates), STI52 (three isolates), ST395 (one isolate), ST707 (one isolate), ST2434 (one isolate) and ST3248 (three isolates) (see Supplementary material, Data SI). Four isolates represented new STs. The II ST88 isolates were all MRSA and will be described elsewhere (Kpeli et al., manuscript in preparation).

Read-mapping of the 49 genomes against the Sa_aus0325 reference sequence produced a 2.2-Mbp core genome with 100 36 I SNPs. A maximum likelihood phylogeny was inferred from pairwise comparisons of these SNPs (Fig. 2). Among the 13 STI5 isolates, ten were isolated from one health centre, including from the hand of an HCW, patients and equipment, corresponding to results from spa typing and giving support to the conclusion that transmission events were ongoing within this health centre. The three isolates from patient A were of the same ST type (ST3248) and between them had SNP differences of 29 bp, $5 \mathrm{I}$ bp and 34 bp (see Supplementary material, Data S2) also corresponding with the spa typing results discussed above. This small number of SNP differences points to the isolates spreading from a common source.

We then inferred the resistome of each isolate from the WGS data (see Materials and methods). Antibiotic resistance genes coding for resistance to $\beta$-lactams (blaZ), chloramphenicol (cat and catpC22I), trimethoprim ( $\mathrm{d} f \mathrm{rG}$ ), methicillin (mecA), quinolone (norA), streptomycin (str) and tetracycline (tetK, tetL and tet $M)$, were identified in $48(98 \%)$, one (2\%), $22(45 \%), 13$

TABLE 2. Comparison of antibiotic resistance between methicillin-resistant and methicillin-susceptible Staphylococcus aureus

\begin{tabular}{|c|c|c|c|}
\hline \multirow[b]{2}{*}{ Antibiotics } & \multicolumn{2}{|l|}{ Resistance rates } & \multirow[b]{2}{*}{ p-value } \\
\hline & $\begin{array}{l}\text { MRSA, } n=31 \text {, } \\
n(\%)\end{array}$ & $\begin{array}{l}\text { MSSA, } n=70 \text {, } \\
n(\%)\end{array}$ & \\
\hline $\begin{array}{l}\text { Amikacin } \\
\text { Gentamicin } \\
\text { Tetracycline } \\
\text { Chloramphenicol } \\
\text { Cotrimoxazole } \\
\text { Ampicillin } \\
\text { Clindamycin } \\
\text { Erythromycin } \\
\text { Rifampicin } \\
\text { Streptomycin } \\
\text { Vancomycin }\end{array}$ & $\begin{array}{l}3(9.6) \\
3(9.6) \\
24(77.4) \\
24(77.4) \\
10(32.3) \\
29(93.5) \\
6(19.4) \\
8(25.8) \\
11(35.5) \\
5(16.1) \\
11(35.5)\end{array}$ & $\begin{array}{l}2(2.8) \\
5(7.1) \\
33(47.1) \\
43(61.4) \\
22(31.4) \\
60(85.7) \\
25(35.7) \\
15(21.4) \\
35(50.0) \\
21(30.0) \\
15(21.4)\end{array}$ & $\begin{array}{l}0.167 \\
0.698 \\
0.005 \\
0.170 \\
1.000 \\
0.335 \\
0.110 \\
0.617 \\
0.200 \\
0.217 \\
0.147\end{array}$ \\
\hline
\end{tabular}


FIG. 2. Whole genome phylogeny of sequenced isolates. Maximum likelihood phylogeny of whole genome sequencing isolates. The tree was rooted in the midpoint. Numbers in nodes indicate support values in the form of proportions of bootstrap pseudoreplicates. Branches with support values $>55 \%$ are collapsed. Greencoloured strains are from Health centre $A$ and the blue ones are from Health centre $B$. The red-coloured circles represent isolates from patient A, yellow-coloured circles represent methicillin-resistant Staphylococcus aureus and the violet circles show isolates from a healthcare worker, patients and equipment in Health centre $B$.

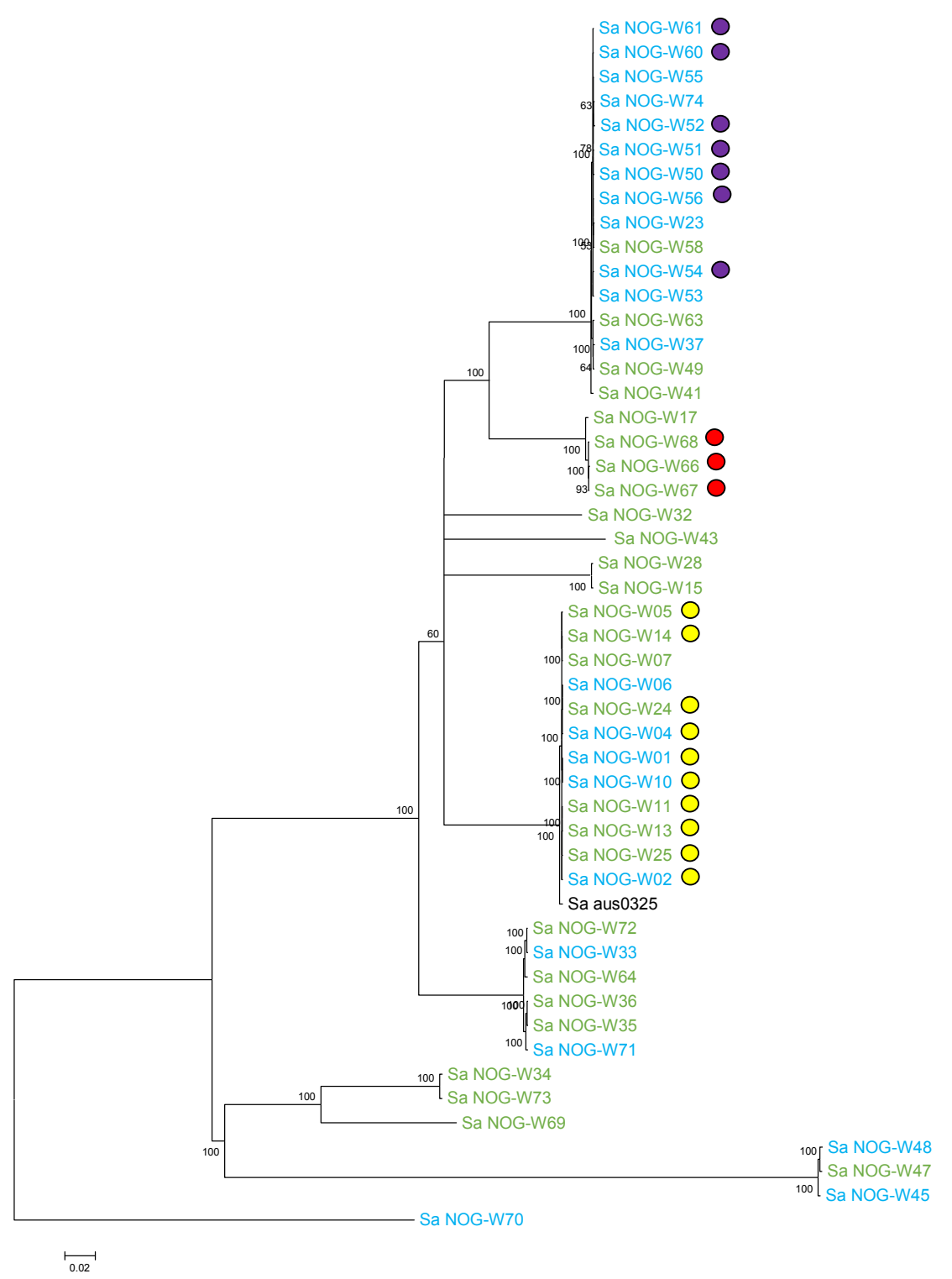

(25.5\%), 13 (25.5\%), 49 (100\%), I2 (24.5\%), eight (16.3\%), 12 $(24.5 \%)$ and $12(24.5 \%)$ of the 49 isolates, respectively (see Supplementary material, Data SI). We further investigated the rpoB gene of rifampicin-resistant strains and identified two known amino acid substitutions $\mathrm{H} 48 \mathrm{IN}$ and I527M implicated in rifampicin resistance in seven and one isolate, respectively (see Supplementary material, Data SI). Other mutations were also found within the rifampin resistance-determining (Rif) region of the rifampicin-resistant isolates but further studies are needed to ascertain if these mutations contribute to rifampicin resistance.

Analysis of the topologies of the phylogenies produced by spa typing and WGS shows lots of agreement but also some differences (Fig. 3). Clustering of MRSA isolates from patient $A$ and isolates from facility $B$ from an HCW, the patients dressed by this worker and equipment were both predicted by the two methods. However, though spa typing predicted the clustering of isolate SA_NOG-WI5, which is an MRSA, with other MRSA isolates, this cluster was not confirmed by WGS, which predicted it to cluster with isolate SA_NOG-W28, an MSSA.

\section{Discussion}

This study confirmed healthcare-associated infection (HAl) as a source of wound infection within our study health centres. Our analysis shows that spa typing is useful for predicting transmission patterns in resource-limited settings but that there is also a need for access to low-cost microbial genomics in 


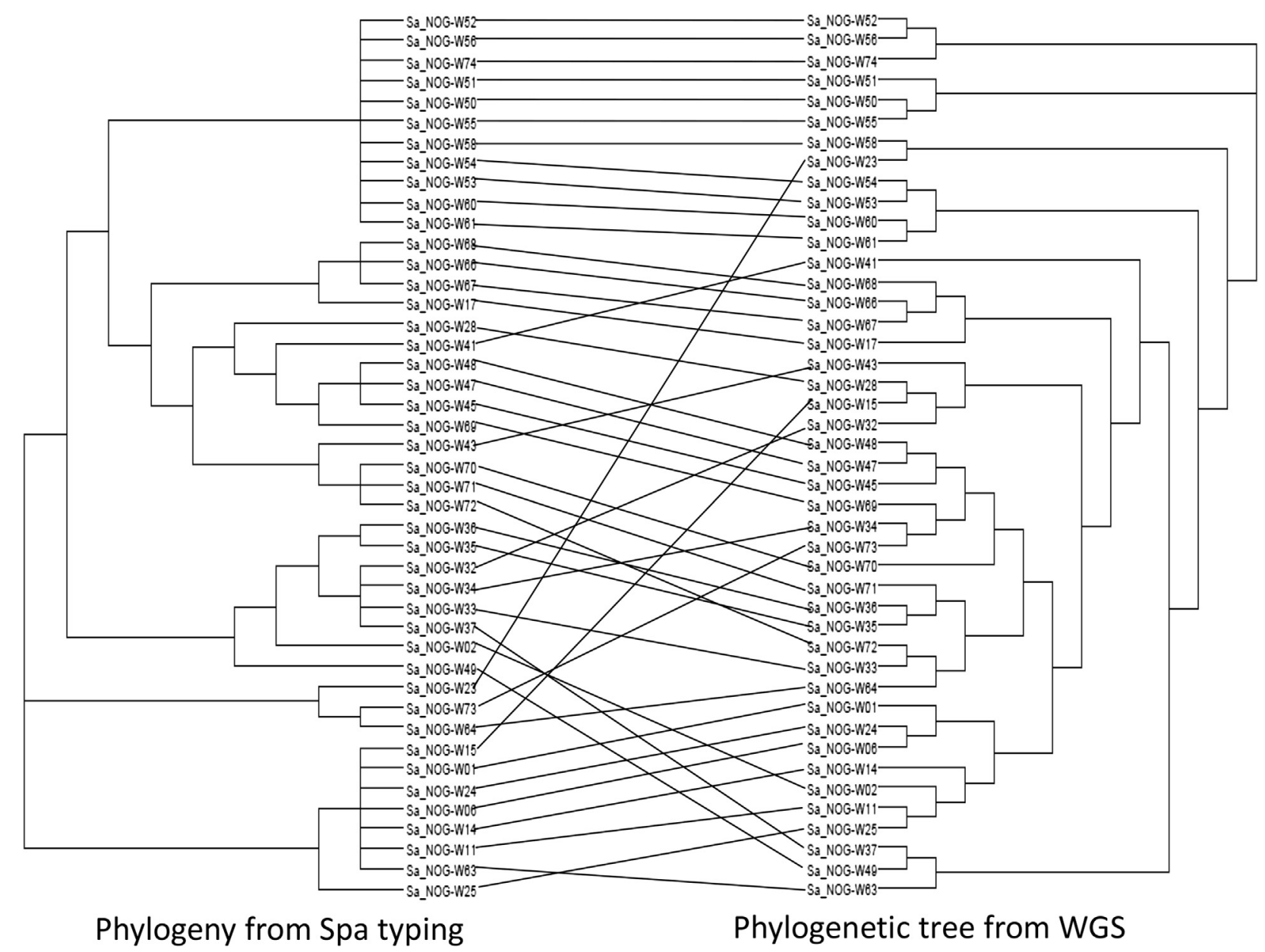

FIG. 3. Tanglegram of spa and whole genome phylogenies. Tanglegram of spa (a) and whole genome sequencing (b) phylogenies produced in dendroscope. Topologies show some agreement between phylogenies but an overall lack of congruence.

developing countries. Genome analysis rapidly revealed widespread antibiotic resistance among the isolates and clearly identified likely transmission clusters.

In previous work [6], we found that wound infection may be a source of healing delay. The findings from our current study implicate the healthcare environment (including healthcare personnel) as possible sources of $S$. aureus infection. From our cluster analysis using spa typing, we inferred three modes of wound infection; two health-facility-related sources through an $\mathrm{HCW}$ and the inanimate environment (Fig. I), and the third source through self infection (Fig. I). This result corresponds with that of previous studies, which have implicated HCW, patients and the inanimate environment in the transmission and subsequent acquisition of S. aureus in healthcare settings [I7]. HAls are known to negatively impact healthcare delivery around the world. Effective infection prevention and control practices, especially compliance with hand hygiene recommendations, will lead to significant reduction in the rate of HAls. Ghana has a policy document to aid the training of HCW in infection prevention and control (www.ghanahealthservice. org/division-scat.php?ghsdid=6\&ghsscid=96). However, a monitoring survey in selected health facilities within the Greater Accra region showed that the compliance level of $\mathrm{HCW}$ to these guidelines is low (Ghana Health Service 2011, Infection Prevention and Control. A survey in Greater Accra, unpublished) with rates below the $70 \%$ recommended by the $\mathrm{WHO}$. Adherence to strict policies supported by periodic training and monitoring of $\mathrm{HCWs}$ is required to ensure compliance with existing infection prevention and control guidelines and to decrease the frequency of HAls.

With regard to the patient-specific clusters, Yeboah-Manu et al. [6] reported that some patients recycle bandages used in wound dressing because of inadequate financial support during their treatment period, and this could result in the transfer of pathogens from one lesion to another. Additionally, wound management in Ghana is influenced to a high degree by local traditional beliefs and practices. Beliefs revolving around the category of people deemed qualified to manage wounds 
affect the behaviour of patients. A recent study from our team (Koka et al. manuscript in preparation) reports that in many communities, pregnant women and nursing mothers are seen as unqualified to manage wounds and in cases where HCWs fall into this category, patients resort to the re-dressing of their wounds after the $\mathrm{HCW}$ has dressed them. This could also lead to the transfer of pathogenic organisms into the lesions as patients do not observe proper aseptic procedures during wound redressing. Hence, there is a need to counsel patients to adhere to the biomedical wound care and management practices to reduce or avoid self infection of their lesions.

This study was limited by not performing a thorough investigation of other body sites of the patients where $S$. aureus is known to exist as a normal flora. An exhaustive investigation should have included culture of samples from other areas of the patients such as the skin, hand and anterior nares of the nasal cavity to compare between strains from these sites and the wounds. The study may also have been affected by the Hawthorne effect at Facility $A$ and this could account for no $S$. aureus isolates from sampling the environment and HCWs at this facility.

Two methods were employed to arrive at our conclusion of HAls; the single gene locus DNA sequence-based marker spa typing and WGS. As revealed from our analysis, phylogenies from both methods predicted similar clusters. Whereas WGS reveals variability across the whole genome and is able to discriminate down to single nucleotide differences, spa typing looks at genetic variability at a single locus between 200 and $600 \mathrm{bp}$ in length. Spa typing is less expensive and demanding in terms of infrastructure and expertise and has a shorter turnaround time compared with WGS. Our results show that the level of discrimination of spa typing is adequate to guide infection control and also supports its use in epidemiological studies. However, the lack of congruence between the two methods probably indicates that spa typing lacks resolution to be able to differentiate between highly genetically related isolates. This partial sequencing technique also cannot reveal the finer genetic details that accumulate during the evolution of bacterial populations. Therefore, although spa typing is useful for the prediction of transmission events in resource-limited settings, where access to the newer, more expensive and more advanced WGS technologies is limited, we should be looking for ways to implement low-cost microbial genomics in these countries because the rich data obtained from pathogen genomes can be used to make well-informed decisions to control the spread of disease.

Another important finding from this study is the high genetic diversity and PVL-positivity among the isolates. This agrees with existing knowledge on the genetic diversity and PVL prevalence among African S. aureus isolates [18-20]. It also supports the assertion that Africa is a PVL endemic continent with high prevalence of PVL being a distinguishing trait of African S. aureus isolates [19] compared with Asia, Europe and the USA [2I]. Additionally, we identified $\mathrm{t} 355$ as one of the most prevalent spa types. This correlates with studies in Ghana and other African countries that identified this spa type as one of the most widespread, hence suggesting it to be widely established and distributed in Africa [18,22,23].

Clinical S. aureus isolates are known to be agr positive [24] and this is also evidenced from our results with 79 (78.2\%) isolates having this locus. However, 22 (21.8\%) isolates were defective for agr function. Previous research suggests that agrdefective mutants can interact with agr-positive variants during clinical infection [24]. These agr-defective mutants play an important role in persistent infection by forming thicker biofilms compared with agr-positive isolates [25]. This phenomenon might play a role in delayed wound healing, which we observed among patients within our study health centres [6].

Antibiotic resistance rates from both phenotypic and resistome investigations confirm the increasing prevalence of drug resistance in Ghana [26]. Thirty-one isolates (30.7\%) were confirmed as MRSA. These data are consistent with the recognized fact that Africa has an intermediate prevalence of MRSA, usually between $25 \%$ and $50 \%$ [27]. Of the 31 isolates, seven showed susceptible phenotypes, though the mecA gene was detected in molecular analysis. Phenotypically susceptible mecA-positive clones have been reported by various research groups [28,29]. The MRSA phenotype is regulated by two genes mecl-mecRI. The induction of MRSA expression by this system is, however, very slow and may render some isolates with the mecA gene phenotypically susceptible. The existence of such strains represents a hidden reservoir for transmission of the methicillin-resistance gene in any environment. In many resource-limited settings, clinicians mostly rely on the results of culture and drug susceptibility testing to guide treatment of patients and most laboratories are also not equipped for molecular testing of organisms. As these clones can only be detected through molecular analysis, they will be reported as susceptible organisms, which will lead to treatment failure.

Of equal concern is the observed high vancomycin resistance of $26 \%$, which is comparable to vancomycin-resistant rates of between $57.7 \%$ [30] and $89 \%$ [3I] that have been reported by other studies from West Africa.

\section{Conclusion}

Our findings indicate that healthcare-associated transmission contributes to wound infection and call for periodic training in infection prevention and control practices to prevent the occurrence of epidemics of nosocomial MRSA. 


\section{Acknowledgements}

We are grateful to the following collaborators and health centres for their involvement in the study: Dr Albert Paintsil, Korle-Bu Teaching Hospital; Mr Lamptey, Obom Health Centre, Obom and Nurses at the Buruli Ward, Ga-West Municipal Hospital, Amasaman.

\section{Appendix A. Supporting information}

Additional Supporting Information may be found in the online version of this article at http://dx.doi.org/10.1016/j.nmni.2016. 07.00I.

\section{Transparency declaration}

The authors have no conflicts of interest to declare.

This work was supported by the Stop Buruli initiative of the UBS Optimus foundation and the Volkswagen Foundation. The funders had no role in study design, data collection and interpretation, or the decision to submit the work for publication. Part of this work was presented at the European Congress on Tropical and Medical Health (ECTMIH) 2015 in Basel, Switzerland from 6 to 10 September 2015 with the abstract subsequently published in Tropical Medicine and International Health 20:68, September, 2015; the Ghana Biomedical Convention (GBC) 2015 University of Ghana, Legon from the 29 to 31 July 2015 and the 26th European Congress of Clinical Microbiology and Infectious Diseases (ECCMID), the annual yearly congress of the European Society of Clinical Microbiology and Infectious Diseases (ESCMID), in Amsterdam from 9 to 12 April 2016.

\section{References}

[I] Robson MC. Wound infection. A failure of wound healing caused by an imbalance of bacteria. Surg Clin North Am 1997;77:637-50.

[2] Bowler PG, Davies B]. The microbiology of acute and chronic wounds. Wounds 1999;1 1:72-9.

[3] Bowler PG, Duerden Bl, Armstrong DG. Wound microbiology and associated approaches to wound management. Clin Microbiol Rev 2001;14:244-69.

[4] Brook I, Randolph JG. Aerobic and anaerobic bacterial flora of burns in children. J Trauma 1981;21:313-8.

[5] Kluytmans J, van Belkum A, Verbrugh H. Nasal carriage of Staphylococcus aureus: epidemiology, underlying mechanisms, and associated risks. Clin Microbiol Rev 1997; 10:505-20.

[6] Yeboah-Manu D, Kpeli GS, Ruf MT, Asan-Ampah K, Quenin-Fosu K, Owusu-Mireku E, et al. Secondary bacterial infections of buruli ulcer lesions before and after chemotherapy with streptomycin and rifampicin. PLoS Negl Trop Dis 2013;7:e2191.

[7] Clinical, Laboratory, Standards, Institute. Performance standards for Antimicrobial Susceptibility Testing: Twenty fourth Informational Supplement MI00-S24. Wayne, PA, USA: CLSI; 2014.

[8] Ruppitsch W, Indra A, Stoger A, Mayer B, Stadlbauer S, Wewalka G, et al. Classifying spa types in complexes improves interpretation of typing results for methicillin-resistant Staphylococcus aureus. J Clin Microbiol 2006:44:2442-8.

[9] Votintseva AA, Fung R, Miller RR, Knox K, Godwin H, Wyllie DH, et al. Prevalence of Staphylococcus aureus protein A (spa) mutants in the community and hospitals in Oxfordshire. BMC Microbiol 20|4; I4:63.

[10] AL-T F, Brunel AS, Bouzinbi N, Corne P, Banuls AL, Shahbazkia HR. DNAGear-a free software for spa type identification in Staphylococcus aureus. BMC Res Notes 2012;5:642.

[II] Staden R, Beal KF, Bonfield JK. The Staden package, 1998. Methods Mol Biol 2000;| 32:1 I5-30.

[12] Oliveira DC, de Lencastre H. Multiplex PCR strategy for rapid identification of structural types and variants of the mec element in methicillin-resistant Staphylococcus aureus. Antimicrob Agents Chemother 2002;46:2|55-61.

[13] Deurenberg RH, Vink C, Driessen C, Bes M, London N, Etienne J, et al. Rapid detection of Panton-Valentine leukocidin from clinical isolates of Staphylococcus aureus strains by real-time PCR. FEMS Microbiol Lett 2004:240:225-8.

[14] Shopsin B, Mathema B, Alcabes P, Said-Salim B, Lina G, Matsuka A, et al. Prevalence of agr specificity groups among Staphylococcus aureus strains colonizing children and their guardians. J Clin Microbiol 2003:41:456-9.

[15] Price MN, Dehal PS, Arkin AP. FastTree 2-approximately maximumlikelihood trees for large alignments. PLoS One 2010;5:e9490.

[16] Huson DH, Scornavacca C. Dendroscope 3: an interactive tool for rooted phylogenetic trees and networks. Syst Biol 2012;61:|06|-7.

[17] Gastmeier P, Sohr D, Geffers C, Behnke M, Daschner F, Ruden H. Mortality risk factors with nosocomial Staphylococcus aureus infections in intensive care units: results from the German Nosocomial Infection Surveillance System (KISS). Infection 2005;33:50-5.

[18] Egyir B, Guardabassi L, Sorum M, Nielsen SS, Kolekang A, Frimpong E, et al. Molecular epidemiology and antimicrobial susceptibility of clinical Staphylococcus aureus from healthcare institutions in Ghana. PLoS One 2014;9:e897|6.

[19] Breurec S, Fall C, Pouillot R, Boisier P, Brisse S, Diene-Sarr F, et al. Epidemiology of methicillin-susceptible Staphylococcus aureus lineages in five major African towns: high prevalence of Panton-Valentine leukocidin genes. Clin Microbiol Infect 201 I; 17:633-9.

[20] Schaumburg F, Kock R, Friedrich AW, Soulanoudjingar S, Ngoa UA, von Eiff C, et al. Population structure of Staphylococcus aureus from remote African Babongo Pygmies. PLoS Negl Trop Dis 20II;5:el I50.

[2I] von Eiff C, Friedrich AW, Peters G, Becker K. Prevalence of genes encoding for members of the staphylococcal leukotoxin family among clinical isolates of Staphylococcus aureus. Diagn Microbiol Infect Dis 2004:49:157-62.

[22] Egyir B, Guardabassi L, Esson J, Nielsen SS, Newman MJ, Addo KK, et al. Insights into nasal carriage of Staphylococcus aureus in an urban and a rural community in Ghana. PLoS One 2014;9:e96II9.

[23] Schaumburg F, Ngoa UA, Kosters K, Kock R, Adegnika AA, Kremsner PG, et al. Virulence factors and genotypes of Staphylococcus aureus from infection and carriage in Gabon. Clin Microbiol Infect 2011;17:1507-13.

[24] Traber KE, Lee E, Benson S, Corrigan R, Cantera M, Shopsin B, et al. agr function in clinical Staphylococcus aureus isolates. Microbiology 2008; 154:2265-74

[25] Kong KF, Vuong C, Otto M. Staphylococcus quorum sensing in biofilm formation and infection. Int J Med Microbiol 2006;296:133-9. 
[26] Newman MJ, Frimpong E, Donkor ES, Opintan JA, Asamoah-Adu A. Resistance to antimicrobial drugs in Ghana. Infect Drug Resist 201 I;4: $215-20$.

[27] Stefani S, Chung DR, Lindsay JA, Friedrich AW, Kearns AM, Westh H, et al. Meticillin-resistant Staphylococcus aureus (MRSA): global epidemiology and harmonisation of typing methods. Int J Antimicrob Agents 20I2;39:273-82.

[28] Petinaki E, Kontos F, Maniatis AN. Emergence of two oxacillinsusceptible mecA-positive Staphylococcus aureus clones in a Greek hospital. J Antimicrob Chemother 2002;50:1090-I.
[29] Cuirolo A, Canigia LF, Gardella N, Fernandez S, Gutkind G, Rosato A, et al. Oxacillin- and cefoxitin-susceptible meticillin-resistant Staphylococcus aureus (MRSA). Int J Antimicrob Agents 201 I;37:178-9.

[30] Olayinka BO, Olayinka AT, Onaolapo JA, Olurinola PF. Pattern of Resistance to vancomycin and other antimicrobial agents in staphylococcal isolates in a university teaching hospital. Afr Clin Exp Microbiol 2005;6:46-52.

[31] Onanuga A, Oyi AR, Onoalapo JA. Prevalence and Susceptibility pattern of methicillin resistant Staphylococcus aureus isolates among healthy women in Zaria, Nigeria. Afr J Biotechnol 2005;4:|32 I-4. 


\section{University Library}

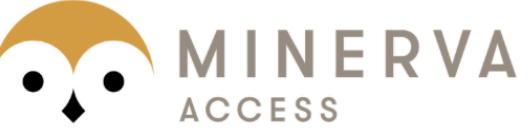

A gateway to Melbourne's research publications

Minerva Access is the Institutional Repository of The University of Melbourne

\section{Author/s:}

Kpeli, G;Darko Otchere, I;Lamelas, A;Buultjens, AL;Bulach, D;Baines, SL;Seemann, T;Giulieri, S;Nakobu, Z;Aboagye, SY;Owusu-Mireku, E;Pluschke, G;Stinear, TP;YeboahManu, D

Title:

Possible healthcare-associated transmission as a cause of secondary infection and population structure of Staphylococcus aureus isolates from two wound treatment centres in Ghana.

Date:

2016-09

\section{Citation:}

Kpeli, G., Darko Otchere, I., Lamelas, A., Buultjens, A. L., Bulach, D., Baines, S. L., Seemann, T., Giulieri, S., Nakobu, Z., Aboagye, S. Y., Owusu-Mireku, E., Pluschke, G., Stinear, T. P. \& Yeboah-Manu, D. (2016). Possible healthcare-associated transmission as a cause of secondary infection and population structure of Staphylococcus aureus isolates from two wound treatment centres in Ghana.. New Microbes New Infect, 13, pp.92-101. https://doi.org/10.1016/j.nmni.2016.07.001.

Persistent Link:

http://hdl.handle.net/11343/256414

License:

CC BY 